\title{
Erratum to: Serum level of soluble (pro)renin receptor is modulated in chronic kidney disease
}

\author{
Kazu Hamada Yoshinori Taniguchi $\cdot$ Yoshiko Shimamura $\cdot$ Kosuke Inoue $\cdot$ Koji Ogata \\ Masayuki Ishihara $\cdot$ Taro Horino $\cdot$ Shimpei Fujimoto $\cdot$ Takashi Ohguro $\cdot$ Yukio Yoshimoto • \\ Mika Ikebe $\cdot$ Kenji Yuasa $\cdot$ Eri Hoshino $\cdot$ Tatsuo Iiyama $\cdot$ Atsuhiro Ichihara $\cdot$ Yoshio Terada
}

Published online: 26 September 2013

(C) Japanese Society of Nephrology 2013

\section{Erratum to: Clin Exp Nephrol \\ DOI 10.1007/s10157-013-0803-y}

The original version of this article unfortunately contained errors.
In Table 1, in the first column, for the line "(P)RR", the unit should be "ng/ml".

In Figs. 1, 2, 3, 4, 5, 7, and 8, on the vertical axes, the unit for "soluble (P)RR" should be "ng/ml".

In Fig. 6, on the vertical axis, the unit for "prorenin" should be "ng/ml".

The online version of the original article can be found under doi: 10.1007/s10157-013-0803-y.

K. Hamada · Y. Taniguchi · Y. Shimamura - K. Inoue ·

K. Ogata $\cdot$ M. Ishihara $\cdot$ T. Horino $\cdot$ S. Fujimoto .

Y. Terada $(\varangle)$

Department of Endocrinology, Metabolism and Nephrology,

Kochi University School of Medicine, Kohasu, Oko-cho,

Nankoku, Kochi 783-8505, Japan

e-mail: terada@kochi-u.ac.jp

T. Ohguro $\cdot$ Y. Yoshimoto

Department of Internal Medicine, Kochi Red Cross Hospital,

Kochi, Japan

M. Ikebe $\cdot$ K. Yuasa

Department of Urology and Internal Medicine,

Kochi-Takasu Hospital, Kochi, Japan

E. Hoshino - T. Iiyama

Clinical Trial Center, Kochi University School of Medicine,

Kochi, Japan

A. Ichihara

Department of Endocrinology and Hypertension, Tokyo

Women's Medical University, Tokyo, Japan 